\title{
Sex Discriminatory Effectiveness Using Combinations of Permanent Teeth
}

\author{
Stanley M. Garn, Patricia E. Cole, Robert L. Wainwright, and Kenneth E. Guire \\ Center for Human Growth and Development, University of Michigan, Ann Arbor, \\ Michigan 84109 USA
}

Since males and females consistently differ in tooth-crown size, it is practical to employ crownsize diameters for "sexing" recent or archaeological material (Ditch and Rose, $A m J$ Phys Anthrop, 37:61-64, 1972; Corrucarin and Henderson, $J$ Dent Res, 55:713, 1976). The question next arises as to the maximum extent of sex discrimination that is possible with crownsize measurements and indices as well as the minimum number of teeth that are effective in stepwise discriminant analysis.

We have made use of mesiodistal and buccolingual diameters of the right permanent teeth of 109 white boys and 95 white girls. Odontometric dimensions were measured on an optical digitizing device, the OPTOCOM (VAN DER LINDEN et al, J Dent Res, 51:1100, 1972; Moyers et al, Standards of Human Occlusal Development 1976, pp 7-10). The measurements, averaged from multiple casts of each individual, were also converted into a mesiodistal-to-buccolingual ratio for each tooth. The resulting values were included in a discriminant analysis (AFIFI and

Received for publication October 5, 1976.

Accepted for publication October 29, 1976.

This study was supported in part by Grant DE03610 from the National Institutes of Health.
AZEN, Statistical Analysis: A Computer Oriented Approach, 1972, pp 252-254).

The sexes were systematically better discriminated by the mesiodistal diameters than the buccolingual crown diameters and by the simple crown dimensions as compared with the calculated ratio. Using the mesiodistal diameters of 14 teeth (excepting M3), up to $86 \%$ of individuals were correctly classified as to sex. Using the best possible combination of dimensions, up to $87 \%$ of boys and girls were correctly sexed by the stepwise discriminant analysis. Maximum discriminatory effectiveness was achieved with as few as six teeth (upper and lower canines, lower second molar, upper and lower lateral incisors, and upper second premolars) using the mesiodistal diameter alone.

These sex assignments, using measurements obtained under optimal conditions and on unworn dentitions, are based on the same individuals included in the stepwise analyses and thus represent maximum discriminatory effectiveness. However, the results suggest that the teeth alone, and relatively few teeth, can be used to "sex" forensic specimens and victims of mass disasters with a degree of success equal to or exceeding that commonly reported for the entire skeleton.

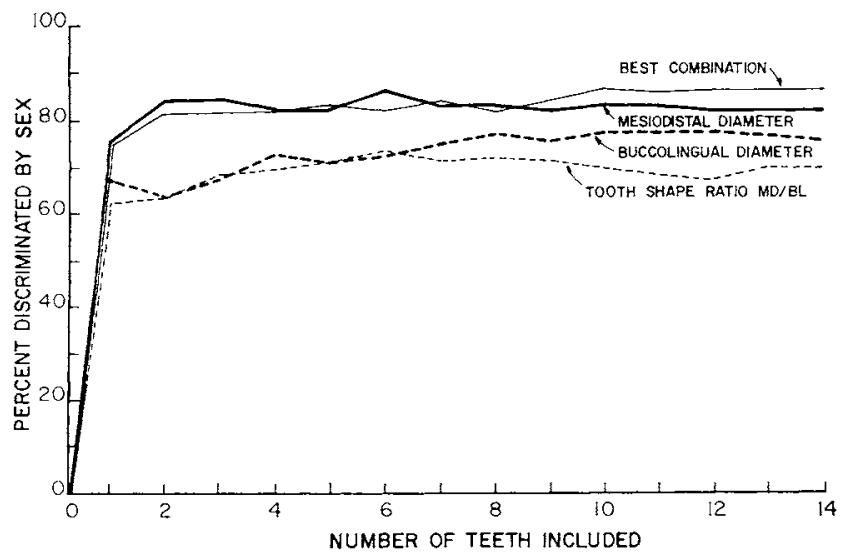

Fig-Extent of sex discrimination employing increasing numbers of teeth (abscissa) and for different crown-size dimensions and combinations (indicated by separate lines). As shown, sex discrimination of over $85 \%$ of subjects can be effected with as few as 6 permanent teeth. 